\section{A) Check for updates}

Cite this: Nanoscale, 2020, 12, 23570

\title{
Sputter deposition of highly active complex solid solution electrocatalysts into an ionic liquid library: effect of structure and composition on oxygen reduction activity $\dagger$
}

\author{
Alba Garzón Manjón, (D) *a Tobias Löffler, ${ }^{b}$ Michael Meischein, ${ }^{c}$ Hajo Meyer, ${ }^{c}$ \\ Joohyun Lim,,$^{\mathrm{a}}$ Valerie Strotkötter, ${ }^{\mathrm{c}}$ Wolfgang Schuhmann, (D) ${ }^{\mathrm{b}}$ Alfred Ludwig (D) $* \mathrm{c}$ \\ and Christina Scheu (D) *a
}

\begin{abstract}
Complex solid solution electrocatalysts (often called high-entropy alloys) present a new catalyst class with highly promising features due to the interplay of multi-element active sites. One hurdle is the limited knowledge about structure-activity correlations needed for targeted catalyst design. We prepared $\mathrm{Cr}-$ $\mathrm{Mn}-\mathrm{Fe}-\mathrm{Co}-\mathrm{Ni}$ nanoparticles by magnetron sputtering a high entropy Cantor alloy target simultaneously into an ionic liquid library. The synthesized nanoparticles have a narrow size distribution but different sizes (from $1.3 \pm 0.1 \mathrm{~nm}$ up to $2.6 \pm 0.3 \mathrm{~nm}$ ), different crystallinity (amorphous, face-centered cubic or bodycentered cubic) and composition (i.e. high $\mathrm{Mn}$ versus low $\mathrm{Mn}$ content). The $\mathrm{Cr}-\mathrm{Mn}-\mathrm{Fe}-\mathrm{Co}-\mathrm{Ni}$ complex solid solution nanoparticles possess an unprecedented intrinsic electrocatalytic activity for the oxygen reduction reaction in alkaline media, some of them even surpassing that of Pt. The highest intrinsic activity was obtained for body-centered cubic nanoparticles with a low $\mathrm{Mn}$ and Fe content which were synthesized using the ionic liquid 1-etyl-3-methylimidazolium bis(trifluoromethylsulfonyl)imide [Emimi][(Tf $\left.)_{2} \mathrm{~N}\right]$.
\end{abstract}

Received 24th October 2020 Accepted 12th November 2020 DOI: 10.1039/d0nr07632e rsc.li/nanoscale excellent candidates for ORR catalysis. ${ }^{1-14}$ In particular, CSS catalysts might have the capability to overcome existing limitations regarding position in volcano plots and scaling relations. ${ }^{6,15}$ However, little is known about structure and composition-activity correlations of CSS catalysts and an increased understanding is a crucial missing piece of information for targeted catalyst design.

Traditional wet synthesis routes are suitable for the synthesis of single-element or binary alloys. ${ }^{16,17}$ The synthesis of single-phase CSSNPs demands a technique with great control over the nucleation process down to the atomic scale, which can be achieved by combinational co-sputtering into ionic liquids (ILs), ${ }^{18,19}$ which offers an almost unlimited flexibility for the used pure elements. ILs are excellent dispersion media to control size, structure and composition of CSSNPs. ${ }^{20,21}$ Additionally, ILs act as a media for growth and as stabilizer for highly stable colloidal solutions, ${ }^{22}$ devoid of any other chemical stabilizers which could affect the CSSNPs catalytic properties.

CSSNPs compositions can be defined by co-sputtering of several elements simultaneously from individual targets or by using alloy targets. ${ }^{18,21}$ In addition, the desired phase, crystallinity and size of the CSSNPs can be modulated by means of post-annealing treatment. ${ }^{21}$ Co-sputtering is a high-through- 
put deposition technique, which allows for screening, but also upscaling of the production of CSSNPs seems feasible. ${ }^{23}$

In an earlier work $^{1}$ we used 1-butyl-3-methylimidazolium bis (trifluoromethylsulfonyl)imide [Bmim] $\left[(\mathrm{Tf})_{2} \mathrm{~N}\right]$ as IL to synthesize binary, quaternary and one type of quinary CSSNPs. The quinary NPs were in the as-grown state amorphous and had an average composition of $\mathrm{Cr}_{39} \mathrm{Mn}_{2} \mathrm{Fe}_{12} \mathrm{Co}_{24} \mathrm{Ni}_{23}{ }^{21}$ and they showed similarly high electrocatalytic ORR activity as PtNPs prepared by sputtering into the same IL. ${ }^{1}$ However, other ILs were not used for the synthesis of quinary NPs until now. We used before the concept of sputtering into a library of ILs with different anions and cations for the formation of pure $\mathrm{Ag}$ NPs. ${ }^{20}$ An influence of the IL's molecular structure on the NPs size and morphology was found.

These results motivated us to synthesize $\mathrm{Cr}-\mathrm{Mn}-\mathrm{Fe}-\mathrm{Co}-\mathrm{Ni}$ NPs by sputtering into a library of eight different ILs. Identical process parameters as ensured by simultaneous preparation as well as cleaning steps allow to compare the effect of different ILs on the CSSNPs formation and their behavior during the ORR. Aberration-corrected (scanning) transmission electron microscopy ((S)TEM) complemented with energy-dispersive X-ray spectroscopy (EDS) are used to characterize the CSSNPs. The results show that the CSSNPs size, crystal structure, and composition depend on the used IL, and accordingly variations in the electrocatalytic ORR activities are observed. Using this approach, we identify several CSSNPs with a high intrinsic activity even higher than Pt.

\section{Results}

During the CSSNPs synthesis, an identical flux of neutral sputtered atoms from the alloy target arrives at the surface of IL library (Fig. 1). The IL library contains eight IL, from which five have the same base cation (1-butyl-3-methylimidazolium) but different base anions, while the other three have the same base anion (bis(trifluoromethylsulfonyl)imide) but different base cations. Their molecular structure is displayed in Fig. $1 \mathrm{~b}$.

We choose these 8 different ILs as we want to study systematically the effect on the molecular chain, lengths and structure. In addition, they have a high purity and stability and low water content. The different properties of the ILs are expected to lead to differences in NP properties.

We investigated the intrinsic electrocatalytic activity of the individual CSSNPs after NP immobilization at etched carbon nanoelectrodes from IL as demonstrated and established in an earlier work. ${ }^{1}$ The voltammetric activity curves are summarized in Fig. 2 and indeed, a strong effect of IL-dependent NP properties on activity can be observed. The group of IL 1, IL 6, IL 7, and IL 8 presents the highest catalytic performance (with the order IL $6>$ IL $7>$ IL $8>$ IL 1) followed by the group of IL 3, IL 4 and IL 5. CSSNPs synthesized with IL 2 have an intrinsic activity which lies between the two groups and is similar to the benchmark Pt NPs. Please note that all curves including Pt seem to be shifted towards higher overpotentials due to the low mass loading of the isolated NPs. As described in more

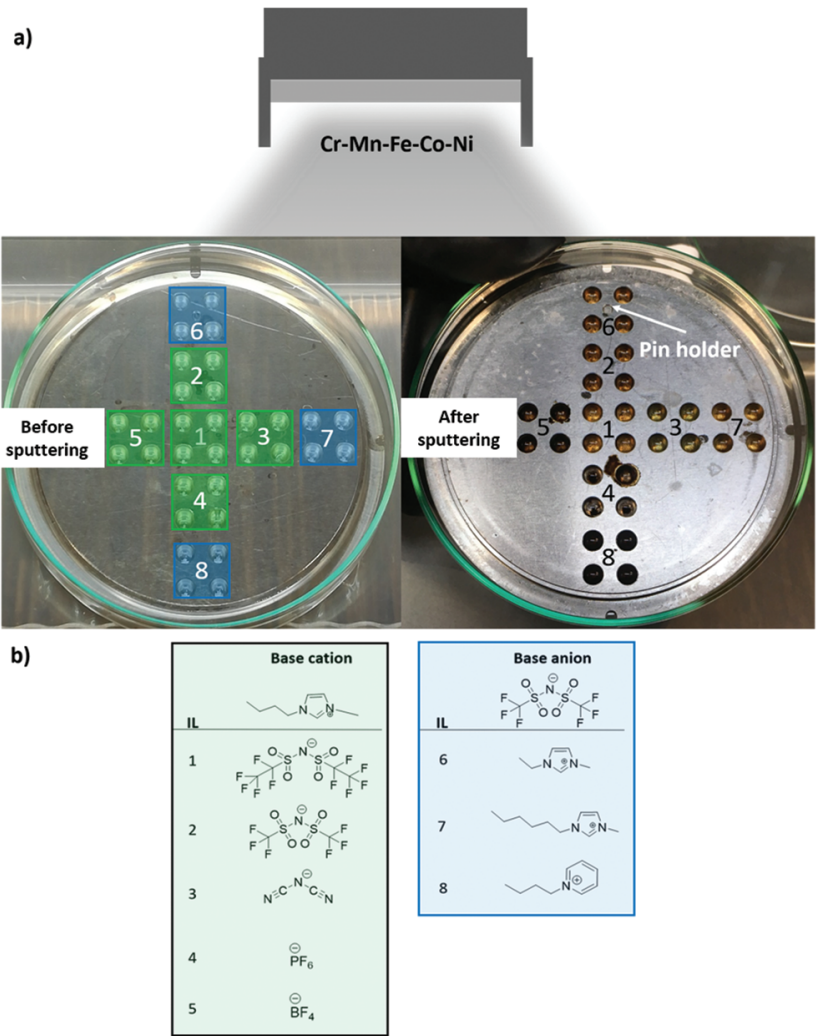

Fig. 1 (a) Schematic of sputtering into a library of 8 ILs to obtain a library of CSSNPs. From the $\mathrm{Cr}-\mathrm{Mn}-\mathrm{Fe}-\mathrm{Co}-\mathrm{Ni}$ alloy target atoms are sputtered simultaneously and deposit in different ILs contained in a cavity holder. Photographs before and after sputtering show a color change after sputter synthesis in the ILs. (b) Molecular structures of the 8 ILs divided in two subgroups according on the common cation and anion.

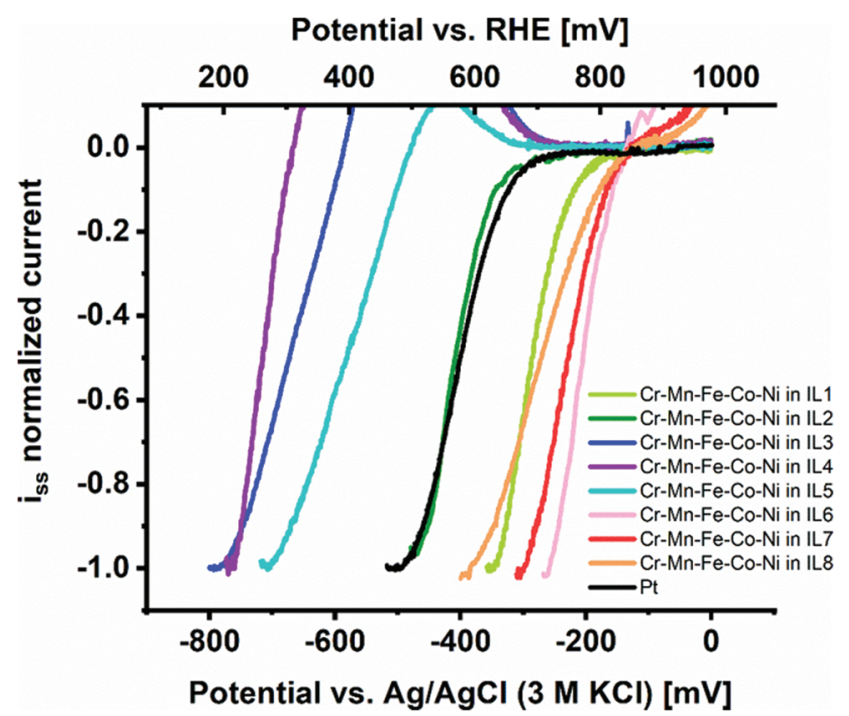

Fig. 2 Comparison of intrinsic ORR activity in alkaline media of CSSNPS immobilized on etched carbon nanoelectrodes from ILs 1 to 8 in comparison to Pt NPs. 
detail in the ESI of ref. 24, a mass loading reduced by two orders of magnitude and constant background noise level provided by the carbon electrode and a Tafel slope of $80 \mathrm{mV} \mathrm{dec}^{-1}$ would imply a visible Pt current exceeding the background current at $160 \mathrm{mV}$ higher overpotential. This finding is in accordance with ref. 1, where we also used IL 2 to produce one amorphous quinary NP system $\left(\mathrm{Cr}_{39} \mathrm{Mn}_{2} \mathrm{Fe}_{12} \mathrm{Co}_{24} \mathrm{Ni}_{23}\right)$, which outperformed binary and quaternary CSSNPs (an in situ phase transformation video showing the transition from the amorphous to the crystalline state is added in the ESI $\dagger$ ). In the present work, the most active CSSNPs show considerably higher intrinsic activity than the benchmark Pt NPs, showcasing the high potential of NP property optimization to exploit the paradigm changing concept of CSS catalysts.

Note that a completely stable "blank" electrode signal cannot be maintained, implying that a slight overcorrection is performed by subtracting the electrode polarization curve prior to NP immobilization. ${ }^{25}$ This effect causes an "oxidative bump" at about $700 \mathrm{mV}$ vs. reversible hydrogen electrode (RHE) where usually the current increase of the blank electrode becomes visible. For the active samples (CSSNPs in IL 1, IL 2, IL 6, IL 7 and IL 8) this effect does not play a role since they reach the maximum current already at lower overpotentials. For the less active samples (CSSNPs in IL 3, IL 4 and IL 5 ), however, a deviation at low currents is obtained, which is counteracted once the kinetic CSSNP current starts to dominate at higher overpotentials and the influence of the carbon instability becomes insignificant. Such a bump is also observed when subtracting two consecutive blank electrode cycles without any NPs attached and, therefore, it is not attributed to any NP property. All polarization curves were normalized by the plateau current of the first current wave, which provides a mass loading independent information about the position of the most favorable adsorption peak and thus, the potential in intrinsic activity for this catalyst. ${ }^{24}$

In order to clarify the origin of the differences in the CSSNPs activities, aberration-corrected high resolution (HR) TEM and EDS data acquisition in STEM were required to analyze the size, structure and composition of the CSSNPs at the atomic scale.

The average size of the CSSNPs ranges from $1.3 \pm 0.1 \mathrm{~nm}$ to $2.6 \pm 0.3 \mathrm{~nm}$ for the different ILs (Fig. 3). Histograms for the size distribution were obtained for the different CSSNPs by analyzing up to 20 HRTEM images. The data are presented in the ESI Fig. S1. $\dagger$

The crystal structure of the CSSNPs could not be determined by selected area diffraction experiments due to the high contribution from the amorphous signal of the carbon-coated TEM grid. Thus, crystal structures were analyzed by fast Fourier transformation (FFT) and the results are shown as insets in Fig. 3. As the CSSNPs were too small to be tilted into a specific zone axis HRTEM images were taken with random orientation of the CSSNPs. The FFT analysis revealed that by choosing the appropriate IL, the CSSNPs can be synthesized in either amorphous, face centered cubic (fcc) or body centered cubic (bcc) state (Fig. 3). We showed earlier that by ex situ
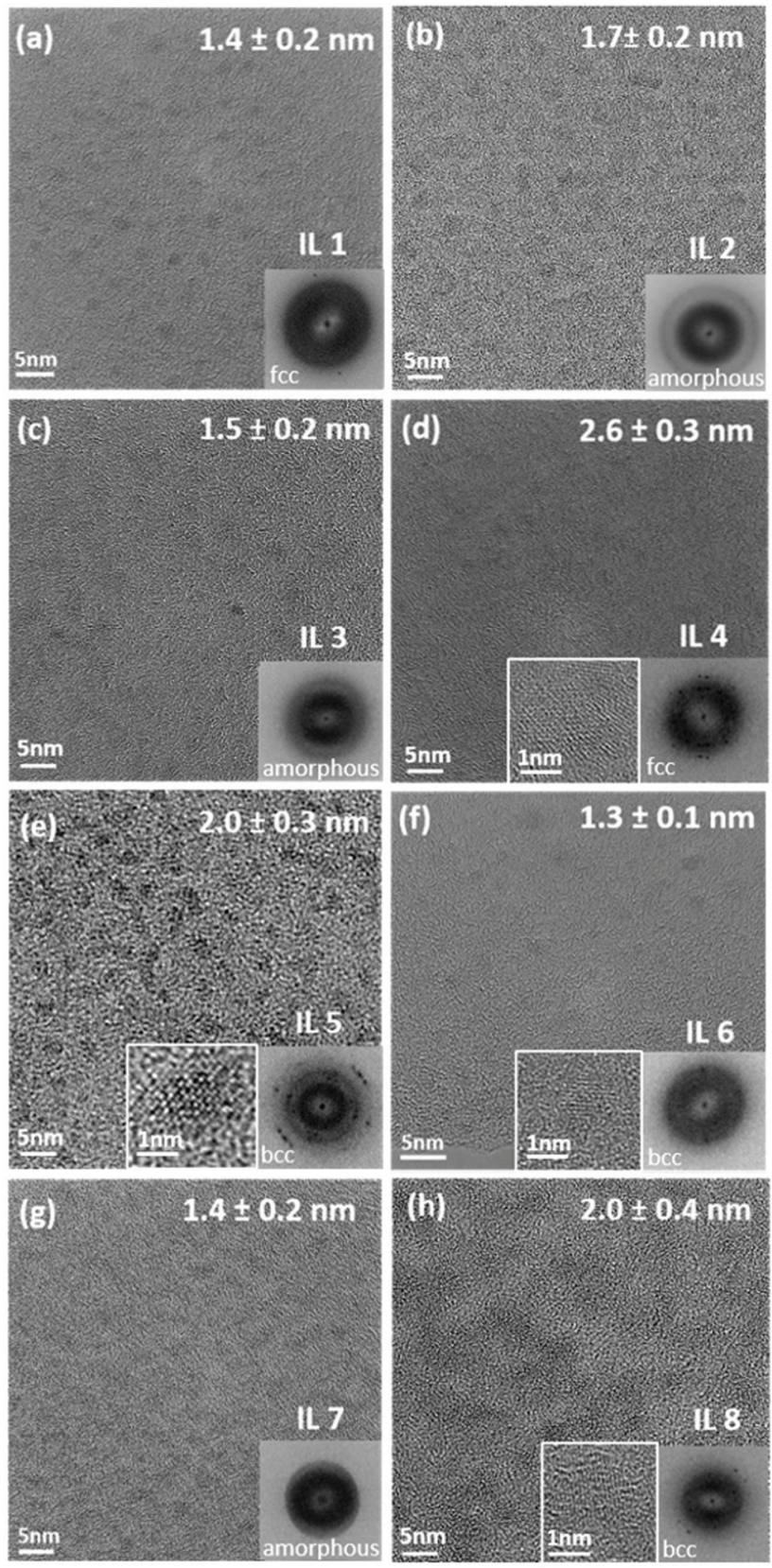

Fig. 3 HRTEM images of CSSNPs sputtered in 8 different ILs: (a) IL 1, (b) IL 2, (c) IL 3, (d) IL 4, (e) IL 5, (f) IL 6, (g) IL 7 and (h) IL 8. The insets show the zoom in on the crystalline CSSNPs and the FFT patterns with the deduced crystal structure. Amorphous, fcc or bcc CSSNPs were found depending on the IL used in the synthesis.

annealing of the initially amorphous NP prepared by DC sputtering, or by high-power impulse magnetron sputtering crystalline NPs can be produced using IL 2. ${ }^{21}$ Here we demonstrate that depending on the IL used we can synthesize CCSNPS with different crystal structures. It should be noted that the different crystal structures are not due to crystallization caused by the electron beam as they were all acquired with similar electron dose rates and at the same $300 \mathrm{keV}$ energy of the incident electron beam. 
To analyze the crystal structure of the different CSSNPs, we used the second reflection due to the following reason. For the fcc structure, the first reflection is $\{111\}$ and the corresponding lattice spacing is $d_{1}=0.208 \mathrm{~nm}$ and the second is $\{022\}$ with $d_{2}$ $=0.180 \mathrm{~nm}$. For bcc, the first reflection is $\{011\}$ corresponding to $d_{1}=0.205 \mathrm{~nm}$ and the second $\{002\}$ with $d_{2}=0.145 \mathrm{~nm}$. Thus, while the difference in the lattice plane spacing $\{111\}$ for fcc and $\{011\}$ for bcc is too small, they can be distinguished by the lattice planes with the higher Miller indices.

CSSNPs synthesized in IL 2, IL 3 and IL 7 do not show a long-range order and are thus considered amorphous. A fcc structure of the CSSNPs was found when using IL 1 and IL 4, while those synthesized with IL 5, IL 6 and IL 8 possess bcc structure. In general, the CSSNPs in the ILs showed a high stability over time and no agglomerates in the colloidal solution were formed after more than one month.

The chemical composition of individual CSSNPs was analyzed by EDS in STEM mode. EDS quantification was achieved using the non-matrix factorization, three main components were identified which were related to the individual CSSNPs, the surrounding IL and the noise/background stemming from the TEM carbon grid. Only the component representative for the individual NPs was quantitatively analyzed of the EDS data by applying principal component analysis. ${ }^{26}$ In order to get compositional values with high accuracy, we determined k-factors from the $\mathrm{Cr}_{18} \mathrm{Mn}_{20} \mathrm{Fe}_{20} \mathrm{Co}_{21} \mathrm{Ni}_{21}$ target. The chemical composition was calculated and averaged for $\sim 20$ CSSNPs. The quantitative analysis revealed that the chemical composition of the individual CSSNPs depends on the IL used in the synthesis. The data together with the observed crystal structure are summarized in Table 1. Fig. 4 shows exemplary the EDS elemental mapping from the CSSNPs synthesized using IL 8 . For these NPs as well as for the ones prepared using the other ILs a homogenous distribution of the five elements was observed.

\section{Discussion}

The synthesis of CSSNPs by sputtering of a $\mathrm{Cr}_{18} \mathrm{Mn}_{20} \mathrm{Fe}_{20} \mathrm{Co}_{21} \mathrm{Ni}_{21}$ target simultaneously in an IL library containing 8 different ILs resulted in different particle sizes, crystal structures, and compositions. These differences emphasize the importance to analyze the ultra-small NPs individually

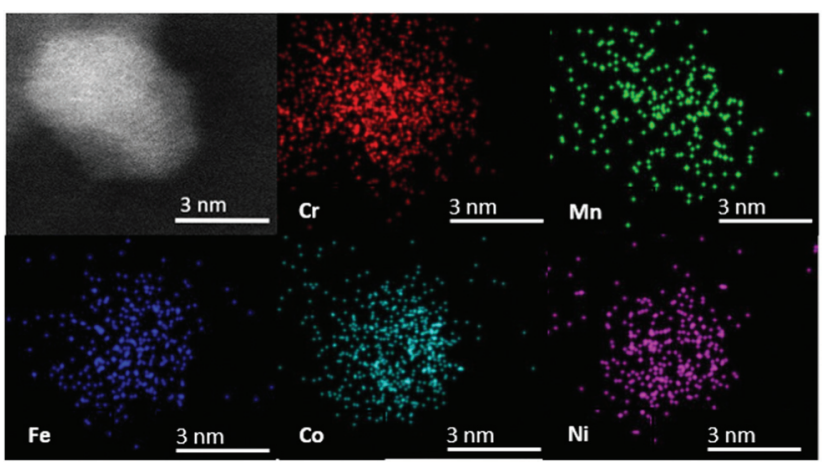

Fig. 4 STEM high angle annular dark field image and corresponding EDS elemental mapping from the CSSNPs synthesized in IL 8. All elements are homogenously distributed.

with aberration corrected (S)TEM and analytical techniques such as EDS. If only average chemical compositions would be considered, a conclusion on structure-properties correlations would be challenging.

The results on the use of different ILs shows the important finding that the molecular structure of the IL influences the size, the composition and the crystal structure as discussed in more detail below. It also demonstrates the capabilities of the synthesis protocol where we kept all parameters identical except for the IL involved which allows to draw the conclusion that the NP properties can be tailored by the choice of the IL.

The chemical composition for the individual CSSNPs, as shown in Table 1, reveals that the sputtered atoms are not interacting equally with the different ILs. If an atom has a sufficient high energy for diffusion inside the IL, it can cluster together with other atoms forming nuclei, which need to overcome a critical nucleus radius to start the growth of CSSNPs. Thus, the composition of individual CSSNPs should be also related locally to the chemical environment and, hence, to the elements of the ILs surrounding the individual nuclei during earlier stages of the nucleation process. ${ }^{27}$

Furthermore, there are three main observations. First, we only observed up to 11 at\% of Mn in the CSSNPs expect for IL 3. From this we can speculated that all the others ILs can act as a chemical barrier for Mn diffusion from the surface at the IL droplet/volume filling into the cavity interior. For the IL 3 the higher content of $\mathrm{Mn}$ can be due to the absence of fluorine groups. ${ }^{28}$ Second, Ni shows for IL 3 a composition of only 3

Table 1 Chemical composition of individual CSSNPs (averaged over 20 particles) without the contribution from the surrounding IL matrix as determined by EDS. In addition, the crystal structure of the CSSNP is given

\begin{tabular}{|c|c|c|c|c|c|c|c|c|}
\hline Elements & $\begin{array}{l}\text { CSSNPs in } \\
\text { IL } 1 \text { (at\%) }\end{array}$ & $\begin{array}{l}\text { CSSNPs in } \\
\text { IL } 2 \text { (at\%) }\end{array}$ & $\begin{array}{l}\text { CSSNPs in } \\
\text { IL } 3 \text { (at\%) }\end{array}$ & $\begin{array}{l}\text { CSSNPs in } \\
\text { IL } 4 \text { (at\%) }\end{array}$ & $\begin{array}{l}\text { CSSNPs in } \\
\text { IL } 5 \text { (at } \%)\end{array}$ & $\begin{array}{l}\text { CSSNPs in } \\
\text { IL } 6 \text { (at } \%)\end{array}$ & $\begin{array}{l}\text { CSSNPs in } \\
\text { IL } 7 \text { (at\%) }\end{array}$ & $\begin{array}{l}\text { CSSNPs in } \\
\text { IL } 8 \text { (at } \%)\end{array}$ \\
\hline $\mathrm{Cr}$ & $37 \pm 1$ & $39 \pm 3$ & $25 \pm 2$ & $32 \pm 2$ & $25 \pm 1$ & $40 \pm 2$ & $37 \pm 1$ & $24 \pm 1$ \\
\hline $\mathrm{Mn}$ & $11 \pm 2$ & $2 \pm 1$ & $19 \pm 1$ & $2 \pm 1$ & $5 \pm 1$ & $6 \pm 1$ & $5 \pm 1$ & $10 \pm 1$ \\
\hline $\mathrm{Fe}$ & $14 \pm 2$ & $12 \pm 2$ & $29 \pm 3$ & $17 \pm 1$ & $16 \pm 2$ & $8 \pm 1$ & $15 \pm 2$ & $19 \pm 1$ \\
\hline Co & $19 \pm 2$ & $24 \pm 2$ & $24 \pm 1$ & $9 \pm 2$ & $25 \pm 2$ & $27 \pm 2$ & $24 \pm 2$ & $23 \pm 1$ \\
\hline $\mathrm{Ni}$ & $19 \pm 3$ & $23 \pm 3$ & $3 \pm 1$ & $40 \pm 2$ & $29 \pm 2$ & $19 \pm 2$ & $19 \pm 3$ & $24 \pm 1$ \\
\hline Crystal structure & fcc & Amorphous & Amorphous & fcc & bcc & bcc & Amorphous & bcc \\
\hline
\end{tabular}


Table 2 Intrinsic ORR activity of CSSNPs sputtered in different ILs together with their related composition trend, particles size and crystal structure. The different colors within the composition denote the molar ratio of the respective element from $40 \%$ (violet) to $1 \%$ (yellow)

\begin{tabular}{lllll}
\hline Activity order & IL & Composition trend & Particles size (nm) & Crystal structure \\
\hline I & 6 & Cr Mn Fe Co Ni & $1.3 \pm 0.1$ & bcc \\
II & 7 & Cr Mn Fe Co Ni & $1.7 \pm 0.2$ & Amorphous \\
III & 8 & Cr Mn Fe Co Ni & $1.5 \pm 0.2$ & Bcc \\
IV & 1 & Cr Mn Fe Co Ni & $1.4 \pm 0.2$ & fcc at \\
V & 2 & Cr Mn Fe Co Ni & $1.7 \pm 0.2$ & Amorphous \\
VI & 5 & Cr Mn Fe Co Ni & $2.0 \pm 0.3$ & bcc \\
VII & 3 & Cr Mn Fe Co Ni & $1.4 \pm 0.2$ & Amorphous \\
VIII & 4 & Cr Mn Fe Co Ni & $2.0 \pm 0.4$ & fcc
\end{tabular}

at $\%$ inside of the CSSNPs. This phenomenon can be due to the specific morphology and charge distribution of the IL 3, which is not favorable for Ni diffusion. Third, Fe has a low concentration $<10$ at $\%$ in IL 6 . The main difference between IL 6 and IL 7 is in their ethyl group form base cation. Thus, it seems that a larger group is needed to allow Fe to enter inside the IL matrix.

The size and the crystal structure are affected by the type of the IL used. The sputtered atoms can diminish the hydrogen bonding network of the IL and lead to a difference in the electrostatic and van der Waals interaction. Furthermore, the Coulomb forces of ILs and the different interactions can lead to various growth mechanisms yielding different crystal structures and sizes, which could be related to the functional group of the IL. ${ }^{28}$

The comparison of ORR activity at a required overpotential to reach -0.5 a.u. on the normalized current scale is summarized in Table 2 and is correlated to composition, particle size and crystallinity. On a first glance it looks like that the group of the most active CSSNPs were obtained irrespective of their crystallinity as they possess a bcc, amorphous or fcc state. However, when comparing the ones with similar sizes (those synthesized with IL 6, IL7 and IL 4) we observe that the one with bcc structure (IL6) has a higher activity then the one in an amorphous state (IL7) and the one with a fcc structure. Thus, our results indicate that the crystalline structure does play, at least, a minor role. The NP size is known to affect the activity due to higher surface areas of smaller particles and different ratios of edge/corner active sites compared to regular lattice plane active sites. ${ }^{29}$ Due to normalizing the current, differences in surface area are already taken into account and the effect on activity would directly displayed by activity trends However, there is no pronounced trend visible, indicating a minor effect compared to the other NP properties.

Four samples show very high activity, namely CSSNPs prepared with IL 1, IL 6, IL 7, and IL 8 (Fig. 3). CSSNPs in IL 2 is following with a small, but clear shift of $120 \mathrm{mV}$ and finally, three samples show a very low activity (CSSNPs in IL 3, IL 4 and IL 5). The different activities can be predominantly related to the different CSSNPs compositions which are determined by the used ILs. Three out of four samples with low activity contain one element with a content of only 1-2 at\% (see Table 2), which might be below the threshold of still preser- ving a CSS phase. In the literature the minimum content in a CSS phase for all elements should be at least 5 at $\% .{ }^{25}$ For the four active samples, each element is at least present with 5 at\% and the NPs are assumed to possess a CSS structure. ${ }^{30}$ Thus, the fluorocarbon from the ILs can play an important role in generating more even content NPs since it was present in all ILs stabilizing the active class of NPs (IL 1, IL 2, IL 6 and IL 8). Comparing the different samples, the composition appears to be most important. This is in line with the current understanding that the presence of a complex solid solution and its constitution at the surface are responsible for the activity. To reach high activities, increased content of $\mathrm{Cr}$ and Co are required. The high activity differences showcase the importance of composition optimization for CSS catalysts.

\section{Conclusions}

Simultaneous sputtering of an alloy target into different ILs created eight colloidal suspensions of CSSNPs. By selection of the stabilizing IL outstanding intrinsic electrocatalytic activity can be achieved. CSSNPs synthesized in IL $1\left([\mathrm{Bmim}]\left[(\mathrm{Pf})_{2} \mathrm{~N}\right]\right)$, IL 6 ([Emimi] $\left.]\left[(\mathrm{Tf})_{2} \mathrm{~N}\right]\right)$, IL $7 \quad\left([\mathrm{Hmim}]\left[(\mathrm{Tf})_{2} \mathrm{~N}\right]\right)$, and IL 8 $\left([\mathrm{BuPy}]\left[(\mathrm{Tf})_{2} \mathrm{~N}\right]\right)$ present even higher catalytic ORR activity than the benchmark Pt NPs, emphasizing the potential of this material class to obtain unprecedented activities induced by the synergy of the multi-element active sites. We investigated the effect of ILs on particle size, crystallinity and the elemental composition by HRTEM and EDS. We revealed that size and crystallinity effect the activity, but the largest contributions is due to the composition which induces large activity differences even though the same set of elements is used throughout. Thus, our work provides improved understanding how to modify the CSSNPs and adapt them to versatile applications as for the ORR.

\section{Experimental}

\section{Synthesis of CSSNPs by sputtering into an IL library ${ }^{20}$}

CSSNPs were synthesized by sputtering in the following ILs (IoLiTec-Ionic Liquids Technologies): (1) IL 1: 1-butyl-3-methylimidazolium bis(perfluoroethylsulfonyl)imide $[\mathrm{Bmim}]\left[(\mathrm{Pf})_{2} \mathrm{~N}\right]$, 
purity > 98\%, halides < $250 \mathrm{ppm}$, water $60 \mathrm{ppm}$. (2) IL 2: 1-butyl-3-methylimidazolium bis(trifluoromethylsulfonyl) imide $[\mathrm{Bmim}]\left[(\mathrm{Tf})_{2} \mathrm{~N}\right]$, purity $>99 \%$, halides $<100 \mathrm{ppm}$, water 51 ppm. (3) IL 3: 1-butyl-3-methylimidazolium dicyanamide $[$ Bmim $]\left[(\mathrm{CN})_{2} \mathrm{~N}\right]$, purity $>98 \%$, halides $<2 \%$, water $1402 \mathrm{ppm}$. (4) IL 4: 1-butyl-3-methylimidazolium hexafluorophosphate $[$ Bmim $]\left[\mathrm{PF}_{6}\right]$, purity $>99 \%$, halides $<100 \mathrm{ppm}$, water $150 \mathrm{ppm}$. (5) IL 5: 1-butyl-3-methylimidazolium tetrafluoroborate $[\mathrm{Bmim}]\left[\mathrm{BF}_{4}\right]$, purity $>$ 99\%, halides $<100 \mathrm{ppm}$, water $109 \mathrm{ppm}$. (6) IL 6: 1-etyl-3-methylimidazolium bis(trifluoromethylsulfonyl)imide [Emimi][(Tf $\left.)_{2} \mathrm{~N}\right]$, purity > 99\%, halides < 100 ppm, water 59 ppm. (7) IL 7: 1-hexyl-3-methylimidazolium bis(trifluoromethylsulfonyl)imide $[\mathrm{Hmim}]\left[(\mathrm{Tf})_{2} \mathrm{~N}\right]$, purity > 99\%, halides < 50 ppm, water 64 ppm. (8) IL 8: 1-butylpyridinium bis(trifluoromethylsulfonyl)imide [BuPy] $\left[(\mathrm{Tf})_{2} \mathrm{~N}\right]$, purity $>$ 99\%, halides < 100 ppm, water 61 ppm. The molecular structure of the different ILs are displayed in Fig. 1.

\section{Sputter deposition ${ }^{21}$}

The sputter process was performed in a magnetron sputter system (AJA POLARIS-5, AJA International) with 1.5 inch diameter cathodes and a DC power supply (DC-XS 1500 from AJA International Inc., North Scituate). Before synthesis the cavity holder was cleaned by ultrasonication for $30 \mathrm{~min}$ each in isopropanol and acetone. A lid was used to expose only 36 cavities. The cavities were filled with the different ILs containing a volume of $40 \mu \mathrm{L}$ per cavity ${ }^{20}$ under Ar gas atmosphere with a purity of $99.9999 \%$. Four cavities were filled with identical IL to increase the total IL volume (Fig. 1). Before sputtering, the ILs were evacuated for three days in the sputter chamber to remove air and moisture until a pressure of $1.7 \times 10^{-4} \mathrm{~Pa}$ was reached. An alloy target was used for sputtering, with a composition of $\mathrm{Cr}_{18} \mathrm{Mn}_{20} \mathrm{Fe}_{20} \mathrm{Co}_{21} \mathrm{Ni}_{21}$, as analyzed by inductively coupled plasma mass spectrometry (ICP-MS), and a purity of 99.95\%. Sputtering was performed at $30 \mathrm{~W}(312 \mathrm{~V}, 95 \mathrm{~mA})$ for $2 \mathrm{~h}$ with rotation of the substrate holder of 30 rotations per minute and an angle of $12^{\circ}$ between the target and the cavity holder. After plasma ignition (1.33 Pa, $20 \mathrm{~W}$ and 2 min precleaning step prior to sputtering) the Ar pressure was fixed to $0.5 \mathrm{~Pa}$, the power was set to $30 \mathrm{~W}$ and the shutter in front of the sputter cathode was opened for the desired time. After the sputter process, the MNP/ILs suspensions were collected and stored under Ar atmosphere in a glovebox (oxygen and water content $<0.5 \mathrm{ppm}$ ).

\section{Electrochemical measurements of CSSNPs on nanoelectrodes}

Etched carbon nanoelectrodes were obtained by preparation of nanopipettes using laser pulling (Sutter Instruments P-2000) of quartz glass capillaries (Sutter Instruments, outer diameter $1.2 \mathrm{~mm}$, inner diameter $0.9 \mathrm{~mm}$ ). Two capillaries with a conically-shaped end with opening between $100 \mathrm{~nm}$ and $250 \mathrm{~nm}$ were obtained. These nanopipettes were flushed with a propane/butane gas mixture from Campinggaz and subsequently heated with a torch at the conical end in an Ar counterflow to fill the capillary with a carbon film. In order to increase the electrode surface area, the thin quartz capillary at the tip apex was removed by etching in $5: 1$ buffered hydrofluoric (HF) solution containing 40\% hydrofluoric acid (aq.) (AnalaR NORMAPUR):40\% $\mathrm{NH}_{4} \mathrm{Cl}$ (aq.) (Sigma Aldrich) by immersion of the tip apex for $4 \mathrm{~min}$. Afterwards, the tip apex was immersed into water to remove any contaminants or $\mathrm{HF}$ residues. ${ }^{31}$ Electrochemical measurements were performed in a three-electrode setup comprising a miniaturized Agar Ag/ $\mathrm{AgCl}(3 \mathrm{M} \mathrm{KCl})$ reference electrode, a carbon cloth in a second compartment $(0.1 \mathrm{M} \mathrm{KOH})$ as counter electrode and the etched carbon nanoelectrodes as the working electrode. A potentiostat (pgu-BI 100 from ips-jaissle) was used for activity measurements of the blank carbon electrode as well as after immobilization of CSSNPs in $0.1 \mathrm{M} \mathrm{KOH}$ in a Teflon beaker. Cyclic voltammograms (CVs) were measured in a potential range between $0 \mathrm{mV}$ and $-800 \mathrm{mV}$ vs. $\mathrm{Ag} / \mathrm{AgCl}(3 \mathrm{M} \mathrm{KCl})$. After every three cycles, the electrodes were lifted and immersed back into the electrolyte to invoke convection and air contact of the electrode surface. This procedure was repeated until three consecutive cycles reached a stable response. The last cycle served as "blank electrode" cycle. After immobilization of CSSNPs, three CV cycles were performed, and the last cycle was used as "electrode + MNP response". By subtraction of the blank electrode current, the CSSNPs signal was obtained as described previously. ${ }^{25}$ For each sample, a new nanoelectrode was used and the $\mathrm{KOH}$ solution was exchanged. CSSNPs immobilization, after the "blank electrode" CV, was obtained with the electrodes immersed for $30 \mathrm{~min}$ into a suspension of $35 \mu \mathrm{l}$ of NPs in IL, $300 \mu \mathrm{l}$ pure IL and $300 \mu \mathrm{l} \mathrm{EtOH}$ while applying a potential of $-400 \mathrm{mV}$ vs. $\mathrm{Ag} / \mathrm{AgCl}(3 \mathrm{M} \mathrm{KCl})$. Electrodes and potentiostat were the same as for activity measurements.

\section{TEM characterization of CSSNPs}

TEM characterization of CSSNPs was carried out using two different Titans 80-300 X-FEG (Thermo Fischer Scientific) operated at $300 \mathrm{kV}$, one equipped with an image corrector and the other one with a probe corrector. A metal-oxide-semiconductor (CMOS) camera with $4 \mathrm{k} \times 4 \mathrm{k}$ pixels was used to record TEM images. EDS was carried out in STEM mode using a beam current of $\sim 150 \mathrm{pA}$ and a beam size of $\sim 0.2 \mathrm{~nm}$. For each sample, approximately 100 CSSNPs were studied. The chemical composition was determined by quantifying the EDS data. In order to investigate the crystal structure of the CSSNPs, FFT were calculated from high-resolution (HR)TEM images, each having a size of $33.5 \times 33.5 \mathrm{~nm}^{2}$, averaging over several CSSNPs. Holey carbon-coated Au grids (200 mesh, Plano) were used to prepare TEM samples. An amount of $2.5 \mu \mathrm{L}$ IL for each sample was dropped on the carbon-coated side and left for adhesion for $2 \mathrm{~h}$. Subsequently, dried acetonitrile was used to clean the grid dropwise for $1 \mathrm{~h}$ under $\mathrm{Ar}$ atmosphere. The final grid was stored inside of a vacuum chamber under Ar atmosphere.

\section{Conflicts of interest}

There are no conflicts to declare. 


\section{Acknowledgements}

T. L. acknowledges support by "Fonds der Chemischen Industrie" for granting a PhD scholarship. Prof. Dr Guillaume Laplanche is acknowledged for providing the sputter target. Torsten Schwarz is acknowledge for helpful discussions. C. S. and A. L. gratefully acknowledge funding from the German Science Foundation (DFG) via the project SCHE 634/21-1 and LU1175/23-1. Open Access funding provided by the Max Planck Society.

\section{References}

1 T. Löffler, H. Meyer, A. Savan, P. Wilde, A. Garzón-Manjón, Y. T. Chen, E. Ventosa, C. Scheu, A. Ludwig and W. Schuhmann, Discovery of a Multinary Nobel Metal Free Oxygen Reduction Catalyst, Adv. Energy Mater., 2018, 8, 1802269, DOI: 10.1002/aenm.201802269.

2 H. Qiu, G. Fang, Y. Wen, P. Liu, G. Xie, X. Liu and S. Sun, Nanoporous High-Entropy Alloys for High Stable and Efficient Catalyst, J. Mater. Chem. A, 2019, 7, 6499, DOI: 10.1039/c9ta00505f.

3 T. Dang-Bao, D. Pla, I. Favier and M. Gómez, Bimetallic Nanoparticles in Alternative Solvents for Catalytic Purposes, Catalysts, 2017, 7, 207, DOI: 10.3390/ catal7070207.

4 W. Gang, Current Challenge and Perspective of PGM-Free Cathode Catalysts for PEM Fuel Cells, Front. Energy, 2017, 11, 286-298, DOI: 10.1007/s11708-017-0477-3.

5 K. V. Yusenko, S. Riva, P. A. Carvalho, M. V. Yusenko, S. Arnaboldi, A. S. Sukhikh, M. Han and S. A. Gromilov, First Hexagonal Close Packed High-Entropy Alloy with Outstanding Stability under Extreme Conditions and Electrocatalytic Activity for Methanol Oxidation, Scr. Mater., 2017, 138, 22-27, DOI: 10.1016/j.scriptamat.2017.05.022.

6 T. Löffler, A. Savan, A. Garzón Manjón, M. Meischein, C. Scheu, A. Ludwig and W. Schuhmann, Toward a Paradigm Shift in Electrocatalysis Using Complex Solid Solution Nanoparticles, ACS Energy Lett., 2019, 4, 12061214, DOI: 10.1021/acsenergylett.9b00531.

7 S. Mandegarzad, J. B. Raoof, S. R. Hosseini and R. Ojani, Cu-Pt Bimetallic Nanoparticles Supported Metal Organic Framework-Derived Nanoporous Carbon as a Catalyst for Hydrogen Evolution Reaction, Electrochim. Acta, 2016, 190, 729-736, DOI: 10.1016/j.electacta.2016.01.022.

8 T. A. A. Batchelor, K. Jack, S. H. Winther, J. Rossmeisl, J. K. Pedersen, S. H. Winther and I. E. Castelli, HighEntropy Alloys as a Discovery Platform for Electrocatalysis, Joule, 2018, 3, 1-12, DOI: 10.1016/j.joule.2018.12.015.

9 M. W. Glasscott, A. D. Pendergast, S. Goines, A. R. Bishop, A. T. Hoang, C. Renault and J. E. Dick, Electrosynthesis of High-Entropy Metallic Glass Nanoparticles for Designer Multi-Functional Electrocatalysis, Nat. Commun., 2019, 10, 2650 .
10 P. Xie, Y. Yao, Z. Huang, Z. Liu, J. Zhang, T. Li, G. Wang, R. Shahbazian-Yassar, L. Hu and C. Wang, Highly Efficient Decomposition of Ammonia using High-Entropy Alloy Catalysts, Nat. Commun., 2019, 10, 1-12, DOI: 10.1038/ s41467-019-11848-9.

11 W. Dai, T. Lu and Y. Pan, Novel and Promising Electrocatalyst for Oxygen Evolution Reaction based on MnFeCoNi High Entropy Alloy, J. Power Sources, 2019, 430, 104-111, DOI: 10.1016/j.jpowsour.2019.05.030.

12 J. K. Pedersen, T. A. A. Batchelor, A. Bagger and J. Rossmeisl, High-Entropy Alloys as Catalysts for the $\mathrm{CO} 2$ and CO Reduction Reactions, ACS Catal., 2020, 10, 21692176, DOI: 10.1021/acscatal.9b04343.

13 Y. Yao, Z. Huang, P. Xie, S. D. Lacey, R. J. Jacob, H. Xie, F. Chen, A. Nie, T. Pu, M. Rehwoldt, D. Yu, M. R. Zachariah, C. Wang, R. Shahbazian-Yassar, J. Li and L. Hu, Carbothermal Shock Synthesis of High-EntropyAlloy Nanoparticles, Science, 2018, 359, 1489-1494, DOI: 10.1126/science.aan5412.

14 H. J. Qiu, G. Fang, Y. Wen, P. Liu, G. Xie, X. Liu and S. Sun, Nanoporous High-Entropy Alloys for Highly Stable and Efficient Catalysts, J. Mater. Chem. A, 2019, 7, 6499-6506, DOI: $10.1039 /$ c9ta00505f.

15 A. Kulkarni, S. Siahrostami, A. Patel and J. K. Nørskov, Understanding Catalytic Activity Trends in the Oxygen Reduction Reaction, Chem. Rev., 2018, 118, 2302-2312, DOI: 10.1021 acs.chemrev.7b00488.

16 A. Garzón-Manjón, E. Solano, M. de la Mata, R. Guzmán, J. Arbiol, T. Puig, X. Obradors, R. Yáñez, S. Ricart and J. Ros, Induced Shape Controllability by Tailored Precursor Design in Thermal and Microwave-Assisted Synthesis of Fe3O4 Nanoparticles, J. Nanopart. Res., 2015, 17, 1-11, DOI: 10.1007/s11051-015-3070-x.

17 A. Garzón-Manjón, A. Aranda-ramos, B. Melara-Benítez, I. Bensarghin, J. Ros, S. Ricart and C. Nogués, Simple Synthesis of Biocompatible Stable $\mathrm{CeO} 2$ Nanoparticles as Antioxidant Agents, Bioconjugate Chem., 2018, 29, 23252331, DOI: 10.1021/acs.bioconjchem.8b00300.

18 M. Meischein, A. Garzón-Manjón, T. Frohn, H. Meyer, S. Salomon, C. Scheu and A. Ludwig, Combinatorial Synthesis of Binary Nanoparticles in Ionic Liquids by Cosputtering and Mixing of Elemental Nanoparticles, ACS Comb. Sci., 2019, 21, 743-752, DOI: 10.1021/acscombsci.9b00140.

19 D. König, K. Richter, A. Siegel, A. V. Mudring and A. Ludwig, High-Throughput Fabrication of $\mathrm{Au}-\mathrm{Cu}$ Nanoparticle Libraries by Combinatorial Sputtering in Ionic Liquids, Adv. Funct. Mater., 2014, 24, 2049-2056, DOI: 10.1002/adfm.201303140.

20 H. Meyer, M. Meischein and A. Ludwig, Rapid Assessment of Sputtered Nanoparticle Ionic Liquid Combinations, ACS Comb. Sci., 2018, 2, 243-250, DOI: 10.1021/acscombsci.8b00017.

21 A. Garzón-Manjón, H. Meyer, D. Grochla, T. Löffler, W. Schuhmann, A. Ludwig and C. Scheu, Controlling the Amorphous and Crystalline State of Multinary Alloy Nanoparticles in An Ionic Liquid, Nanomaterials, 2018, 8, 903, DOI: 10.3390/nano8110903. 
22 T. Torimoto, T. Kameyama and S. Kuwabata, Top-Down Synthesis Methods for Nanoscale Catalysts, Nanocatal. Ionic Liq., 2016, 171-205, DOI: 10.1002/9783527693283.ch9.

23 S. Kumari, L. Helt, J. R. C. Junqueira, A. Kostka, S. Zhang, S. Sarker, A. Mehta, C. Scheu, W. Schuhmann and A. Ludwig, High-Throughput Characterization of $\mathrm{Ag}-\mathrm{V}-\mathrm{O}$ Nanostructured Thin-Film Materials Libraries for Photoelectrochemical Solar Water Splitting, Int. J. Hydrogen Energy, 2020, 45, 12037-12047, DOI: 10.1016/j. ijhydene.2020.02.154.

24 T. Löffler, A. Savan, H. Meyer, M. Meischein, V. Strotkötter, A. Ludwig and W. Schuhmann, Design of Complex SolidSolution Electrocatalysta by Correlating Configuration, Adsorption Energy Distribution Patterns, and Activity Curves, Angewandte, 2019, 59, 5844-5850, DOI: 10.1002/ anie.201914666.

25 T. Löffler, P. Wilde, D. Öhl, Y. T. Chen, K. Tschulik and W. Schuhmann, Evaluation of the Intrinsic Catalytic Activity of Nanoparticles without Prior Knowledge of the Mass Loading, Faraday Discuss., 2018, 210, 317-332, DOI: $10.1039 / \mathrm{c} 8 \mathrm{fd} 00029 \mathrm{~h}$.

26 S. Zhang and C. Scheu, Evaluation of EELS Spectrum Imaging Data by Spectral Components and Factors from
Multivariate Analysis, Microscopy, 2018, 67, i133-i141, DOI: 10.1093/jmicro/dfx091.

27 Z. He and P. Alexandridis, Nanoparticles in Ionic Liquids: Interactions and Organization, Phys. Chem., 2015, 17, 18238-18261, DOI: 10.1039/C5CP01620G.

28 S. Wegner and C. Janiak, Metal Nanoparticles in Ionic Liquids, Springer International Publishing, 2017, DOI: 10.1007/s41061-017-0148-1.

29 C. Wang, D. Van Der Vliet, K. C. Chang, H. You, D. Strmcnik, J. A. Schlueter, N. M. Markovic and V. R. Stamenkovic, Monodisperse Pt3Co Nanoparticles as a Catalyst for the Oxygen Reduction Reaction: SizeDependent Activity, J. Phys. Chem. C, 2009, 113, 1936519368, DOI: 10.1021/jp908203p.

30 G. Bracq, M. Laurent-Brocq, L. Perrière, R. Pirès, J. M. Joubert and I. Guillot, The fcc Solid Solution Stability in the Co-Cr-FeMn-Ni Multi-Component System, Acta Mater., 2017, 128, 327336, DOI: 10.1016/j.actamat.2017.02.017.

31 J. Clausmeyer, P. Wilde, T. Löffler, E. Ventosa, K. Tschulik and W. Schuhmann, Detection of Individual Nanoparticle Impacts using Etched Carbon Nanoelectrodes, Electrochem. Commun., 2016, 73, 67-70, DOI: 10.1016/j. elecom.2016.11.003. 